\title{
Aspects of the SMOS Pre-launch Calibration
}

\section{Skou, Niels}

\section{Published in:}

IGARRS 2003 Proceedings of IEEE International Geoscience and Remote Sensing Symp

Link to article, DOI:

10.1109/IGARSS.2003.1294064

Publication date:

2003

Document Version

Publisher's PDF, also known as Version of record

Link back to DTU Orbit

\section{Citation (APA):}

Skou, N. (2003). Aspects of the SMOS Pre-launch Calibration. In IGARRS 2003 Proceedings of IEEE International Geoscience and Remote Sensing Symp (Vol. 2, pp. 1222-1225). IEEE.

https://doi.org/10.1109/IGARSS.2003.1294064

\section{General rights}

Copyright and moral rights for the publications made accessible in the public portal are retained by the authors and/or other copyright owners and it is a condition of accessing publications that users recognise and abide by the legal requirements associated with these rights.

- Users may download and print one copy of any publication from the public portal for the purpose of private study or research.

- You may not further distribute the material or use it for any profit-making activity or commercial gain

- You may freely distribute the URL identifying the publication in the public portal 


\title{
Aspects of the SMOS Pre-launch Calibration
}

\author{
Niels Skou \\ Oersted•DTU, B 348, \\ Technical University of Denmark. \\ DK 2800 Lyngby, Denmark. \\ Phone: (45) 452538 00, Fax: (45) 45931634 \\ e-mail: ns@oersted.dtu.dk
}

\begin{abstract}
A synthetic aperture radiometer system, SMOS, is under development for launch in 2007. The synthetic aperture concept requires calibration activities of novel nature in addition to traditional radiometer calibration exercises. Especially very accurate antenna pattern measurements are an issue.
\end{abstract}

\section{INTRODUCTION}

The SMOS (soil moisture and ocean salinity) mission aims at measuring important geophysical parameters by means of L-band radiometry. Despite the fact that the need for global measurements of soil moisture and ocean salinity was recognized already long ago, and the potential of doing it by radiometry has also been well established for years, no space mission has materialized until now. The reason is of course that L-band radiometers having reasonable spatial resolution have been judged to require unrealistically large antenna structures. However, as the synthetic aperture radiometer concept has matured, systems are now deemed viable, and SMOS is now well into phase-B with a scheduled launch in 2007.

SMOS is a $1.4 \mathrm{GHz}$ system with 72 antenna elements and radiometers mounted on three $4.3 \mathrm{~m}$ long arms as well as on a central hub, that also holds the many correlators - one for each possible pair of antennas. Two of the elements in the central hub are actually connected to very accurate noise injection radiometers (NIR) in order to measure the absolute level of the brightness temperature scene being sensed. As any radiometer system, SMOS requires careful calibration, both on-board utilizing built-in calibration means, as well as pre-launch characterization, which is the subject here.

\section{CALIBRATION ISSUES}

The synthetic aperture concept is based on the fact that by measuring an adequate number of the so-called visibilities, the original brightness temperature map can be found by an inverse Fourier transform. The visibilities are in principle outputs of the correlators operating on the outputs of pairs of antenna + radiometer elements. The visibility function corresponding to a pair of channels 1,2 is shown in the following equation where: $\mathrm{k}$ is Boltzman's constant, $\mathrm{B}_{1,2}$ are the noise bandwidths of the channels, $\mathrm{G}_{1,2}$ are the power gains of the channels, $\left\langle\mathrm{b}_{1} \mathrm{~b}_{2}{ }^{*}>\right.$ is the correlator output, $\Omega_{1,2}$ are the equivalent solid angle of the normalized radiation patterns of

$$
\begin{aligned}
& \mathrm{V}_{1,2}=\mathrm{V}\left(\mathrm{u}_{1,2}, \mathrm{v}_{1,2}\right)=\frac{1 / 2}{\mathrm{k} \sqrt{\mathrm{B}_{1} \cdot \mathrm{B}_{2}} \cdot \sqrt{\mathrm{G}_{1} \cdot \mathrm{G}_{2}}} \cdot\left\langle\mathrm{b}_{1}(\mathrm{t}) \mathrm{b}_{2}{ }^{*}(\mathrm{t})\right\rangle= \\
& \frac{1}{\sqrt{\Omega_{1} \cdot \Omega_{2}}} \cdot \iint \frac{\mathrm{T}_{\mathrm{B}}(\xi, \eta)}{\sqrt{1-\xi^{2}-\eta^{2}}} \cdot \mathrm{F}_{\mathrm{n} 1}(\xi, \eta) \cdot \mathrm{F}_{\mathrm{n} 2}^{*}(\xi, \eta) \\
& \cdot \mathrm{r}_{1,2}-\frac{\mathrm{u}_{1,2} \cdot \xi+\mathrm{v}_{1,2} \cdot \eta}{\mathrm{f}_{\mathrm{o}}} \cdot \mathrm{e}^{-\mathrm{j} 2 \pi\left(\mathrm{u}_{1,2} \cdot \xi+\mathrm{v}_{1,2} \cdot \eta\right)} \mathrm{d} \xi \mathrm{d} \eta
\end{aligned}
$$

the antennas, $T_{B}$ is the brightness temperature scene to be sensed, $(\xi, \eta)$ are the directional cosines, $F_{n 1,2}$ are the normalized radiation patterns of the antennas, and $r_{1,2}$ is the fringe wash function accounting for decorrelation effects. It is seen that to obtain the correct value for the visibility function from the measured correlation, many system parameters have to be accurately known. Especially, it is noticed that the antenna patterns have to be known.

SMOS has a built-in calibration network. Each radiometer has at its input a four-way PIN diode switch to select either of the sources: horizontal antenna port, vertical antenna port, 50 ohm load (uncorrelated noise for correlator calibration and radiometer ambient load calibration point), calibration network (correlated noise in all receivers and radiometer hot calibration point). This means that only the antenna (plus a part of the input switch) is outside the on-board calibration loop. Hence, the antenna patterns (amplitude and phase) must be measured before launch, and they must be assumed to preserve their properties during launch and operation in space.

An important fact is that the antenna elements and the associated radiometers are integrated antenna/receiver units, which makes traditional antenna measurements impossible. The signal from the antenna passes through the input switch, an isolator, two amplifiers, and a filter before it can be monitored on a test port. This means that: 1) The antenna transfer functions cannot be measured independently of a part of the receiver transfer function. This has some influence on the measurement quality bearing in mind that the receiver transfer function depends on items like temperature and supply voltage. This should not present big problems as the temperature inside a radio anechoic chamber is quite constant, and high quality power supplies are readily available. But stability of receiver front ends to better than $0.01 \mathrm{~dB}$ over longer measurement sequences is not necessarily a trivial matter. 2) The receiver front end is designed for very low 
signal levels, which means that the antenna measurements must be carried out at considerably lower levels than normally used. This in turn limits the $\mathrm{S} / \mathrm{N}$ ratio, possibly deteriorating measurement accuracy specifications. Preliminary analysis and test runs indicate no problems, however.

\section{ANTENNA PATTERN MEASUREMENTS}

\section{A. Requirements and Facility}

A comprehensive calibration budget has been established using an end-to-end computer simulator. Of interest here are the requirements to the antenna pattern measurements, which are a voltage pattern amplitude uncertainty of $0.05 \mathrm{~dB}$, and a voltage pattern phase uncertainty of $0.33 \mathrm{deg}$. These are stringent requirements to an antenna measurement setup, but it has been found that a state-of-the-art spherical near field facility like the DTU-ESA facility at the Technical University of Denmark can fulfil the requirements.

Another important requirement to the antenna facility is size. It will be discussed later that it is not necessary to measure the full SMOS with its around $9 \mathrm{~m}$ of span, but anyway antenna parts of substantial size must fit in the facility. The DTU-ESA room is $12 \times 10 \times 8 \mathrm{~m}$ between absorber tips and fulfill the size requirements.

Finally, the room must be a Faraday cage to avoid RFI not only during the delicate antenna measurements at unusually low signal levels, but also to facilitate additional radiometric measurements. The DTU-ESA facility is such a Faraday cage, and the integrity of the shielding has been checked by careful measurements inside the room using a well calibrated L-band radiometer.

\section{B. Measurement Strategy}

Due to the combination of mutual coupling between elements and stringent error requirements, it is of little interest to measure individual element patterns. The elements must be mounted in a structure carefully representing the final SMOS structure - if not the final structure itself. But as mutual coupling only plays a role for elements mounted quite close to each other, it is not necessary to have the full structure be measured at any time - which is fortunate as this would have required a very large antenna range.

The SMOS layout is illustrated in Figure 1.
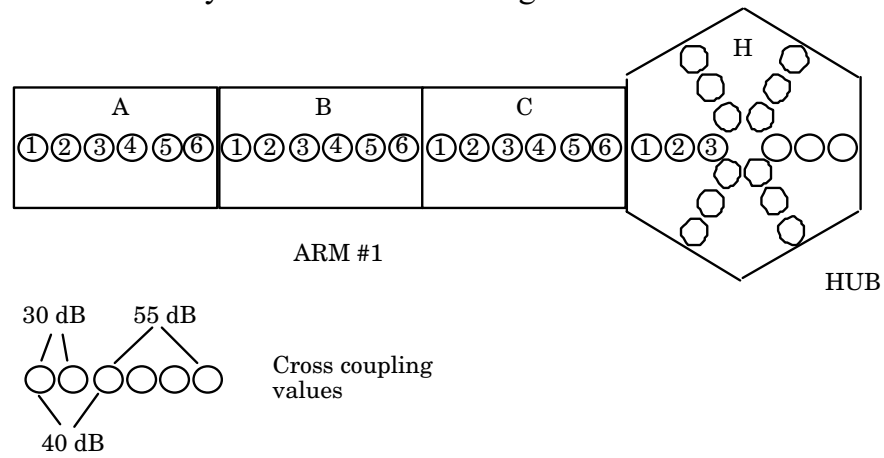

Figure 1: SMOS Layout
Only one arm and the hub is shown, and it is illustrated that preliminary measurements indicate that the coupling between neighboring elements is $30 \mathrm{~dB}$, between two elements with one element in between is $40 \mathrm{~dB}$, and between elements with two elements in between drops to $55 \mathrm{~dB}$.

A coupling between neighboring elements of $30 \mathrm{~dB}$ is certainly respectable, but not enough to ensure that an elements pattern is measured correctly without concern for the neighboring elements, as $30 \mathrm{~dB}$ corresponds to $\pm 0.27 \mathrm{~dB}$ on the patterns. $40 \mathrm{~dB}$ is marginal $( \pm 0.036 \mathrm{~dB}$ influence $)$. But $55 \mathrm{~dB}( \pm 0.015 \mathrm{~dB}$ influence $)$ is a value big enough to ensure that we do not have to consider elements that are further away than two elements. Hence is we consider arm section $\mathrm{B}$ alone we would correctly measure elements B3 and B4 as there are at least two elements present on either side of those. If we take arm section A we would be able to correctly measure A1 - A4. But the remaining elements will only be measurable by joining sections.

When measuring $\mathrm{A}+\mathrm{B}$ we get accurate results for elements $\mathrm{A} 1$ - A6 and B1 - B4. When measuring $\mathrm{B}+\mathrm{C}$ we get accurate results for elements $\mathrm{B} 3$ - B6 and $\mathrm{C} 1$ - $\mathrm{C} 4$. When measuring the hub with arm section $\mathrm{C}$ we get accurate results for several hub elements as well as for elements C3 - C6. By repeating this for all arms it is seen that all elements are measured correctly, with elements B3, B4 and C3, C4 actually being measured twice. The results from the two set of measurements of these elements must be identical thus ensuring that our hypothesis - that elements more than two elements away can be disregarded - holds true.

Since the aforementioned hypothesis is quite important for the whole measurement strategy, it is worth noting that it can actually be checked in greater detail. First, arm section A is measured. Then section B is joined and measurements of the A elements repeated. By comparing the two sets of measurements, it can be seen how far the influence of the B elements propagates into the A section element patterns. This check will actually be done in advance using proper elements in a representative structure.

It should be noted here that if the hypothesis, that we only have to consider the two nearest elements when measuring an element's pattern, should not turn up to hold true, but an additional element must be taken into account, we can still use the same measurement strategy. But now when measuring $\mathrm{A}+\mathrm{B}$ we get accurate results for elements $\mathrm{A} 1$ - A6 and B1 $\mathrm{B} 3$, when measuring $\mathrm{B}+\mathrm{C}$ we get accurate results for elements $\mathrm{B} 4$ - B6 and $\mathrm{C} 1-\mathrm{C} 3$. When measuring the hub with the inner arm section we get accurate results for hub elements as well as for elements C4 - C6. Thus we have all elements measured properly, but no overlap as a surety. If for some reason even more elements are needed to be taken into account, we have a problem that must be further examined.

\section{Number of Measurements and the Time Required}

Following the baseline strategy we have to measure most of the 72 elements once, but B3, B4 and C3, C4 are measured twice, that is 84 elements to be measured. 
Each pattern is measured twice with the measurement distance differing by a quarter of a wavelength. The two measurement results are averaged to minimize the effect of multiple reflections between the probe and the SMOS structure. Since the structure is large this effect cannot be overlooked.

All patterns are measured at 3 frequencies (1404, 1413.5, and $1423 \mathrm{MHz}$ ) and at two probe polarizations, but this is done electronically and automatically as part of the measurement sequence in the DTU - ESA facility. Additionally, each element is measured twice (input switch selecting horizontal or vertical polarization), but this is also done electronically and automatically.

In total we have to carry out $84 \cdot 2=168$ measurements.

Each measurement takes at least 4 hours. In addition to the pure measurement time, some time is spent between measurements to: change connectors from one element to another, change distance between probe and SMOS, check alignments and other issues - on average 1 hour. So, one measurement takes 5 hours.

For a reasonable schedule assuming 2 measurements per day, i.e. a 10 hour working day, 5 days a week this amounts to 17 weeks not including the time for initial set-up (a few days) and major changes like changing from arm measurements to the hub (maybe one day), in total some 18 weeks. This will leave night time for unforeseen problems, but is not a schedule liked by higher level management.

A very ambitious schedule would assume 4 measurements per day, 7 days a week which is 6 weeks plus one week for set-up and interchange. In total 7 weeks. This is a very difficult schedule to realize. No time for unforeseen problems, and work will have to be carried around the clock 7 days a week. This also requires a large amount of skilled personnel.

These schedule considerations are only preliminary, but they stress the fact that the antenna measurements are not only delicate, but also time consuming. A careful planning of the production of elements, the antenna measurements, and whatever other activities with the elements after that, is absolutely necessary.

\section{RADIOMETER CALIBRATION}

SMOS is, after all, a radiometer, and basic radiometer calibration is an issue. The receivers have in addition to their digital outputs for the correlator unit, also a traditionally detected power output. The individual receivers must be calibrated concerning this output. This is not a difficult task as the accuracy requirements are relaxed: the task of these outputs are to be able to measure total system temperature in order to calculate the correlations from the correlation coefficients of the digital correlator. But it is a demanding task is to calibrate the NIRs, which must be done with very good accuracy. Either a complicated variable target is designed and constructed in order to calibrate the NIRs to a fraction of a Kelvin over the full input range; or the NIRs are carefully calibrated at one high brightness temperature and one low brightness temperature, and the linearity of the instrument is checked carefully by proper means. The first method requires a complicated target, the second that the NIRs be linear - or at least nearly linear with a smooth second order transfer function that can be assessed.

A very important parameter concerning radiometer calibration targets is the reflection coefficient. If we assume that we are dealing with a liquid nitrogen cooled target at 77 $\mathrm{K}$ and that the noise temperature being emitted from the radiometer out of the antenna towards the target is $300 \mathrm{~K}$, the radiometer under test will measure the following brightness temperature: $\mathrm{T}_{\mathrm{B}}=77 \mathrm{~K} \cdot \varepsilon+300 \mathrm{~K} \cdot(1-\varepsilon)$ where $\varepsilon$ is the target emissivity. A return loss of $20 \mathrm{~dB}$ corresponds to an emissivity of 0.99 and to an error of $2 \mathrm{~K}$ which is totally unacceptable. But as we pass $30 \mathrm{~dB}$ reflection coefficient, the error drops below $0.2 \mathrm{~K}$, and we approach reasonable figures.

Calibration targets are typically constructed using more or less standard microwave absorbing materials. Typical flat panel absorbers have a reflection coefficient of $20 \mathrm{~dB}$ so they cannot be used for the present purpose. Typical pyramidal absorbers can exhibit a $35 \mathrm{~dB}$ return loss at L-band with a pyramid height of $30 \mathrm{~cm} .35 \mathrm{~dB}$ corresponds to an error of $0.07 \mathrm{~K}$. It can be difficult to assure complete thermal equilibrium of these long pyramids, especially for a target to be operated over a large range of temperatures for example from $77 \mathrm{~K}$ to ambient.

An possible solution to the problem with temperature equilibrium is to use a Brewster angle geometry and much shorter pyramids / wedges as was the case for the SMMR calibration target. This target is outlined in Figure 2.

\section{MICROWAVE CALIBRATION TARGET INVERTED PYRAMID}

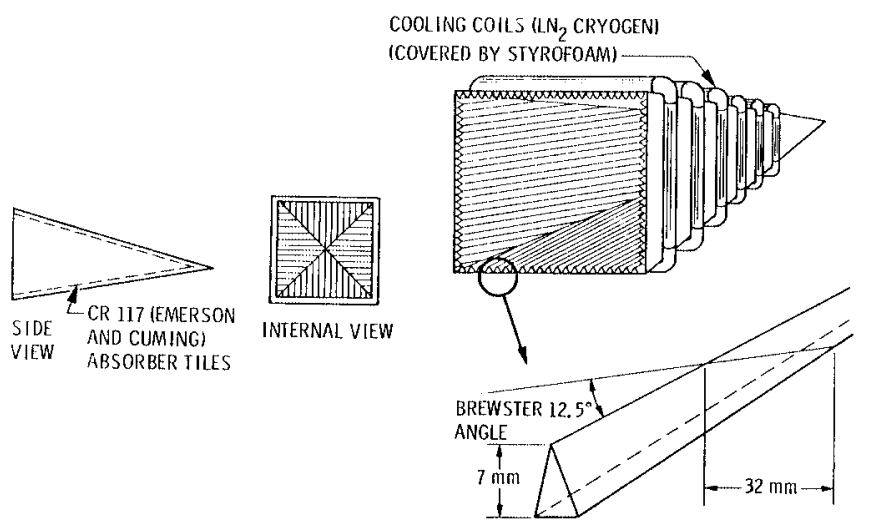

Figure 2: The SMMR target design

The figure shows the variable temperature target that was made in the 70'es by JPL for the calibration of SMMR [3]. The target is fabricated from iron-filled epoxy absorber tiles with a high dielectric constant. The Brewster angle is frequency independent which permits simultaneous calibration at a wide frequency range. The emission temperature of the target can be varied continuously from LN2 temperature to $400 \mathrm{~K}$ by circulating liquid or heated nitrogen gas through the coils attached to the base plates of the tiles. A proportional gas controller maintains the 
temperature at any selected value within the control range with an accuracy of better than $0.5 \mathrm{~K}$. Platinum sensors are embedded in the tiles to measure the temperature to within $0.1 \mathrm{~K}$.

The clear advantage of this system is that there is close contact between the emitting substance and the cooled metal base plates. Despite being able to work even at low microwave frequencies the absorbing material is of thin dimensions meaning better thermal control. The target is very compact and handy - well suited for the SMOS elements.

The target can only be used in very dry conditions due to the risk of ice accumulation on the tile surface in open laboratory environment. The target operates with any pointing geometry. The design must be re-evaluated in view of the requirement for L-band operation.

A simplified, fixed temperature version of the SMMR target for use on the instrument while laying on the floor of a suitable RF shielded room looking upwards is a viable option. The interior of the target is foam filled, while the target itself (without cooling coils) is integrated into the bottom of an insulated metal bucket. The bucket is filled with liquid nitrogen and thus the target walls are in intimate contact with the $77 \mathrm{~K}$ liquid.

Finally, it is noted that at L-band the sky temperature is a very stable, low calibration point only marginally dependent on weather. However, it is well known that the sky brightness temperature is direction dependent, but this effect is well understood and mapped, and can be accounted for. The bucket technique must be used in order to assure that all incident radiation on the antennas originates in the sky, but the bucket, although large in this case, is a simple and straight forward construction. Concern can be expressed as to taking flight hardware outside, but some kind of thin plastic radome should be possible, and the advantage of making a low brightness temperature check of the full instrument should not be underestimated.

\section{OTHER MEASUREMENTS IN THE ANECHOIC CHAMBER}

In addition to the antenna measurements, also other measurements like calibrations and imaging of a range of point targets have to be carried out in a facility like the DTUESA facility. First of all, possible RFI from outside sources is avoided as such a facility is in fact built as a Faraday cage. Secondly, imaging of point targets requires basically free space, i.e. in practice an anechoic chamber, to be sure of representative conditions.

The following measurements can be carried out:

- Calibration of the SMOS radiometers, and final calibration check of the NIR instruments.

- While the instrument is in the floor position, a radiometric measurement of the brightness temperature of the room can be carried out. A noise source mounted at the ceiling can be considered as a point source in the near field of the synthetic antenna, and hence be processed by proper algorithms. As the relation between near field and far field algorithms are well understood, this will serve well as a point source check. The point source could be centered above
SMOS, but it could also be moved around and yield additional information. It is a requirement that the height of the noise source above the SMOS instrument is large enough to ensure that the source is reasonably within the FOV of all the antenna elements (not way down the pattern slopes of some of the antennas).

- It could be considered also to image a more complicated, controlled target in the near field, again using near field image reconstruction algorithms. This might remedy the major problem that it is absolutely impossible to conceive a test setup in which the full instrument images a well controlled, complicated target in the far field. The target in question could be a liquid nitrogen cooled absorber target in a styrofoam container, and a slightly heated absorber for a smaller contrast target.

- Checks of the polarimetric performance are also possible (SMOS has a polarimetric imaging mode). A noise source connected to a single polarized horn antenna, that can be rotated in a controlled fashion around a vertical axis while looking straight down from the ceiling is a viable option. This option, however, yields very large values of the third and fourth Stokes parameters. More realistic signal levels can be generated by using a dual polarized horn and connect the noise source via a power divider directly to for example the $\mathrm{V}$ port. The other part of the signal from the power divider is connected to the $\mathrm{H}$ port via an attenuator. Hence, the level of correlated signal in the orthogonal channel can be adjusted to realistic levels.

\section{CONCLUSIONS}

Taking the SMOS calibration budget as a starting point it has been described how very accurate antenna pattern measurements, and more basic radiometer calibrations have to be carried before launch in order to arrive at calibrated brightness temperature imagery. The voltage pattern measurements can be carried out in a modern state-of-the-art spherical near field antenna range as required concerning amplitude and phase. By a proper measurement strategy it is possible to measure the individual element patterns without the need for a fully deployed SMOS at any given time. The measurements are time consuming and careful planning and scheduling are important. The radiometer calibration exercises require specially developed radiometric targets, and a possible design is discussed.

\section{REFERENCES}

[1] A. Camps, J. Bara, F. Torres, I. Corbella, J. Romeau: "Impact of antenna errors on the radiometric accuracy of large aperture synthesis radiometers", Radio Science, 32, pp 657-668, March 1997

[2] I. Corbella, F. Torres, A. Camps, J. Bara, M. Vall-Ilosera, N. Duffo: "L-band aperture synthesis microwave radiometry: hardware requirements and system performance”, proc. IGARSS'2000

[3] J. M. Stacey, personal communication, JPL, 1980 